\title{
Breaking the Upper Bound of Siloxane Uptake: Metal-Organic Frameworks as an Adsorbent Platform
}

\author{
Ezgi Gulcay (iD †a, Paul lacomi (iD †a, Youngsang Ko ${ }^{b}$, Jong-San Chang ${ }^{b}$, Guillaume Rioland ${ }^{c}$, Sabine Devautour-Vinot (iD a , \\ and Guillaume Maurin (iD) a \\ $\dagger$ These authors contributed equally \\ * E-Mail: guillaume.maurin1@umontpellier.fr
}

Biogas, regarded as a promising renewable energy source, still needs to be upgraded. This calls for the removal of the most prominent contaminants, among others the octamethylcyclotetrasiloxane (D4) molecule. Herein, high throughput computational screening in tandem with synthesis and adsorption testing revealed the hydrophobic Zr-MOF PCN-777 as an optimal D4 adsorbent with record gravimetric $\left(1.8 \mathrm{~g} \mathrm{~g}^{-1}\right)$ and volumetric $\left(0.49 \mathrm{~g} \mathrm{~cm}^{-3}\right)$ uptakes, alongside a reversible and fast adsorption/desorption process, good cyclability and easy regeneration. This MOF was demonstrated to encompass an ideal combination of mesoporous cages and chemical functionality to enable an optimal packing of the siloxane molecules and their efficient removal while maintaining the process highly reversible thanks to moderately high host/guest interactions. This work highlights the efficacy of an integrated workflow for accelerating adsorbent selection for a desired application, spanning the entire pipeline from method validation to computational screening, synthesis and adsorption testing towards the identification of the optimal adsorbents.

\section{Introduction}

Biogas capture from landfill sites or wastewater treatment plants is identified as an appealing strategy to procure a renewable energy fuel, simultaneously promoting a reduction in greenhouse gas emissions and an increase in waste treatment profitability ${ }^{1}$. The use of biogas as an energy green resource critically calls for a substantial increase of its $\mathrm{CH}_{4}$ quality by removing gaseous and vapour impurities resulting from anaerobic digestion processes ${ }^{1}$. One prominent class of biogas impurities are the linear (denoted "L") and cyclic (denoted "D") siloxanes, as degradation by-products of silicone polymers from packaging, construction, cosmetics, and household items ${ }^{2,3}$ This family of molecules is also known to damage subsequent energy recovery systems, e.g. combustion engines, fuel cells and steam reformers, via their decomposition into amorphous silica on heated surfaces that leads to abrasive solid deposits on critical machinery, and to inactivation of gas reforming catalysts ${ }^{4}$. Octamethylcyclotetrasiloxane commonly labelled D4 is the most representative siloxane species present in biogas, which spans from 50 to $70 \%$ of the total siloxane content due to its relatively low water solubility $\left(56 \mu \mathrm{g} \mathrm{I}^{-1}\right)$ and its significant vapour pressure $(196 \mathrm{~Pa} \text { at } 303 \mathrm{~K})^{3-5}$.

Multiple technologies have been proposed to mitigate the presence of siloxanes in biogas outlet streams, including mineral acid/base scrubbing, deep chilling, or iron oxide beds, often working in tandem to remove other impurities ${ }^{6}$. The physisorption-based removal of D4 by porous filters is also a promising alternative, due to its relatively low potential energetic cost, while avoiding the use of environmentally hazardous chemicals $^{7,8}$. A variety of conventional adsorbents has been envisaged for siloxane elimination, including activated carbons ${ }^{9}$, zeolites ${ }^{10}$, and silicas ${ }^{11}$. However, these materials suffer from several drawbacks that limit their use, in particular insuffi-

aCGM, Univ. Montpellier, CNRS, ENSCM, F-34095 Montpellier, France ${ }^{b}$ Research Group for Nanocatalyst (RGN) and Convergent Center for Chemical Process (CCP), Korea Research Institute of Chemical Technology (KRICT), Gajeong-ro 141, Yuseong-gu, Daejeon 34114, South Korea

${ }^{\mathrm{C}}$ Centre National d'Etudes Spatiales, DSO/AQ/LE, 18 Avenue Edouard Belin 31401 Toulouse, Cedex 09, France

Electronic Supplementary Information (ESI) available: one PDF file with all referenced supporting information, CSV file of final screening dataset, all experimental isotherms and PXRD patterns. cient uptake and/or incomplete regeneration under standard conditions. Moreover, downstream biogas commonly contains a proportion of water, which can compete with D4 sorption when using hydrophilic adsorbents ${ }^{6,12}$. Therefore, finding a high capacity adsorbent capable of removing siloxanes under moderate humidity conditions in a reversible manner remains a challenge.

Metal-Organic Frameworks (MOFs) are one of the most recent classes of porous adsorbents. These coordination polymers are built from the assembly of metal nodes and organic multidentate linkers to form architectures of different dimensionality from $1 \mathrm{D}$ to $4 \mathrm{D}^{13-15}$. Their near-infinite diversity, thanks to a wide set of building blocks, has made this class of porous solids promising for applications in gas/vapour adsorption/separation $^{16,17}$, catalysis ${ }^{18}$, and sensing ${ }^{19,20}$ among others. Their high and uniform porosity combined with extensive chemical tunability of their pore walls suggest that MOFs may hold promise as candidates for siloxane adsorption. Insofar only two studies have attempted to investigate the potential of MOFs for D4 removal. Mito-Oka and co-workers ${ }^{21}$ proposed DUT-4(AI) $([\mathrm{Al}(\mathrm{OH})(2,6-\mathrm{ndc}), \mathrm{DUT}$ : Dresden University of Technology), a wine rack-like MOF, as a first potential adsorbent. Although its hydrophobicity makes this MOF attractive for D4 elimination under humidity, its adsorption capacity of $0.15 \mathrm{~g} \mathrm{~g}^{-1}$, estimated through single component by TGA measurements, is rather low and its regeneration can only be achieved at very high temperature, over $523 \mathrm{~K}$, resulting from a high confinement of D4 (kinetic diameter of $8.6 \AA)$ in its channels $(9 \AA \times 9 \AA)$. More recently, MIL-101(Cr) $\left(\mathrm{Cr}_{3} \mathrm{O}(\mathrm{OH})\left(\mathrm{H}_{2} \mathrm{O}\right)_{2}(\mathrm{btc})_{3}\right.$, MIL: Material of Institute Lavoisier), a well-known highly porous MOF incorporating two types of mesoporous cages with diameters of 29 $\AA$ and $34 \AA$ was demonstrated to exhibit a much higher D4 uptake of $0.95 \mathrm{~g} \mathrm{~g}^{-1}$ at $298 \mathrm{~K}$, however its regeneration was only possible upon heating at $423 \mathrm{~K}$ under vacuum ${ }^{22}$. Further, since MIL-101(Cr) is known to be highly hydrophilic ${ }^{23}$ we can expect a substantial drop of its D4 uptake performance even under low-relative humidity. Indeed, neither of these MOFs tested so far combines a large D4 uptake, low-energy regeneration and hydrophobicity to avoid a preferential adsorption of $\mathrm{H}_{2} \mathrm{O}$ over D4 under low to moderate relative humidity.

To date, only a very small number of MOFs has been sampled for this application, and therefore relied on researchers' intuition to identify promising adsorbents. There are, however, 


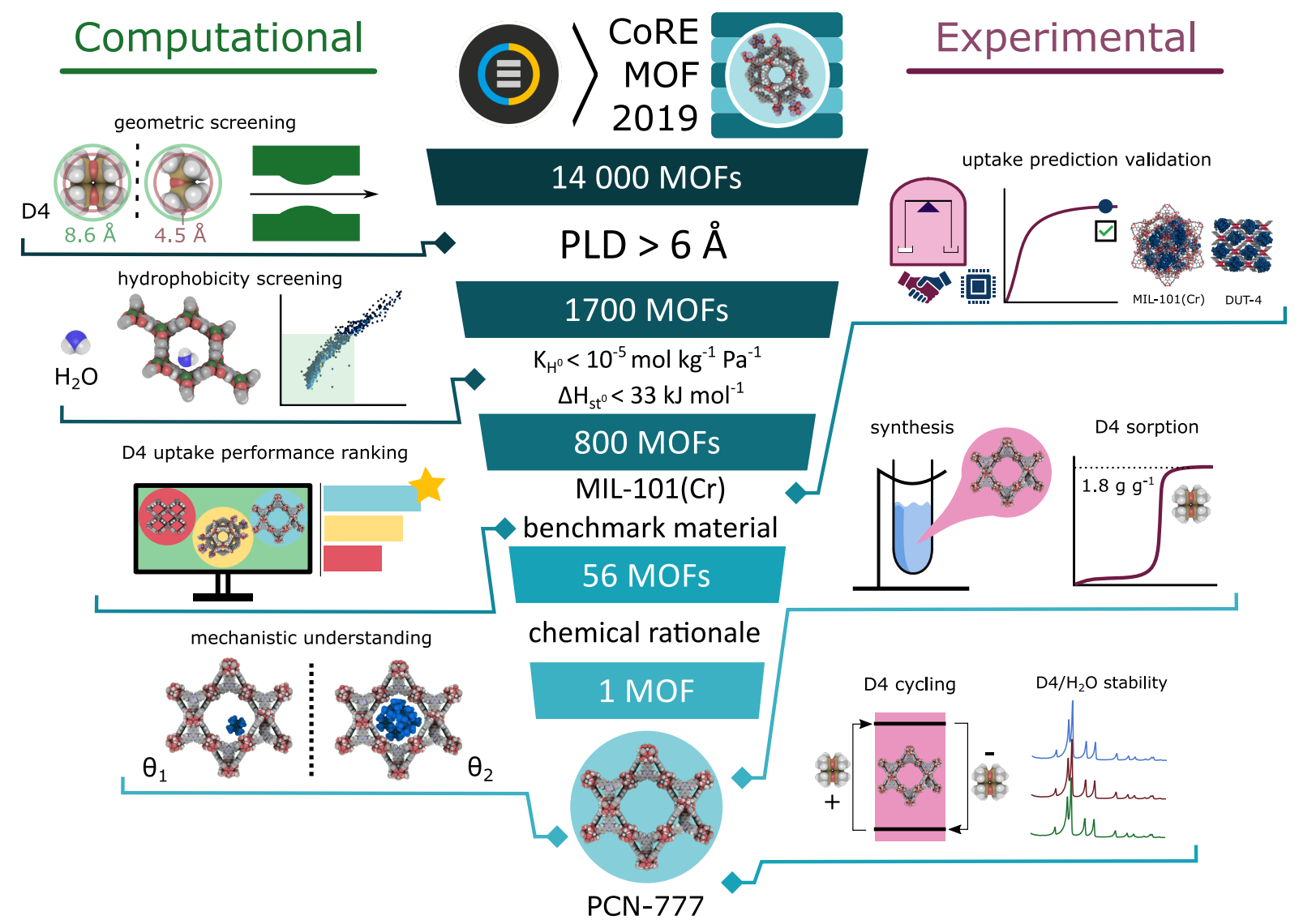

Fig. 1: Workflow of the strategy applied to identify the best MOFs for D4 adsorption, narrowing down candidates from top to bottom through synergistic computational (left) and experimental (right) actions. The final MOF candidate, PCN-777, is highlighted.

a myriad of hydrophobic MOFs that might perform better for D4 adsorption. Since it is unfeasible to individually test the performances of all the existing MOFs, several high throughput computational screening (HTCS) workflows have been devised which identified promising MOFs for diverse adsorption-related applications ${ }^{24-29}$. However, such a computational strategy can only be successful if conducted in strong interplay with a careful analysis of the best-predicted MOF performers in terms of chemical/thermal stability under the target working conditions as well as ease of synthesis/activation. This enables the selection of the MOF candidate with the best overall compromise for further adsorption testing to confirm the expectation.

With this in mind, we herein devise a hand-in-hand computational-experimental strategy whose workflow is summarized in Fig. 1. As a first stage, the CoRE (Computation-Ready, Experimental) MOF 2019 database $^{30}$ was computationally screened with the objective to identify hydrophobic materials showing a D4 uptake higher than the current MOF benchmark, e.g. MIL-101(Cr). Notably, the microscopic models used to describe both MOF and D4 were validated by a good agreement between the simulated D4 uptake and our own experimental data collected on the two MOFs mentioned above, i.e. MIL$101(\mathrm{Cr})$ and DUT-4 $(\mathrm{Al})$. From the top 56 predicted MOF performers, we selected the $\mathrm{Zr}$ carboxylate-based mesoporous PCN-777 (PCN for Porous Coordination Network) for further experimental testing. This MOF was demonstrated to exhibit not only a record D4 uptake $\left(1.8 \mathrm{~g} \mathrm{~g}^{-1}\right)$ to date for a crystalline porous material, but also exceptional cycling and low-energy regeneration without the need for thermal treatment, while its confirmed hydrophobicity strongly suggests a preservation of its adsorption performance under low to moderate relative humidity conditions. An in-depth analysis of the adsorption mechanism further revealed the dominant host-guest interac- tions that control the adsorption of the first D4 molecules and their effective packing in the whole porosity up to saturation.

\section{Methodology}

2.1. Computational methods. We used the CoRE-MOF 2019 database $^{30}$ (over 14000 MOFs), recently updated to remove solvent molecules and disordered structures, to which we also added further 29 well-known MOFs owing to their good chemical/thermal stability and permanent accessible porosity (listed in Table S7 SI). The geometric characterization of MOFs, including pore limiting diameters (PLDs), densities, $\mathrm{N}_{2}$-accessible surface areas (SAs), pore volumes (PVs) and void fractions $(\phi)$, were calculated by Zeo++ software ${ }^{31}$. All Monte Carlo simulations were performed with the RASPA simulation package ${ }^{32}$ Henry coefficients of $\mathrm{H}_{2} \mathrm{O}\left(K_{H, H_{2} O}\right)$ and isosteric enthalpy of adsorption $\left(\Delta H_{s t, H_{2} O}^{0}\right)$ were initially computed at $298 \mathrm{~K}$ for all MOFs using the Widom particle insertion method ${ }^{33}$ These simulations were carried out using $1 \times 10^{5}$ production cycles and $5 \times 10^{4}$ cycles for equilibration. We applied the same Widom insertion method to calculate isosteric enthalpy of adsorption at low coverage for D4 in DUT-4(AI) and PCN-777 with the consideration of $1 \times 10^{6}$ production cycles and $5 \times$ $10^{5}$ steps for equilibration. Continuous fractional component Monte Carlo (CFCMC) simulations ${ }^{34}$ were performed to evaluate the saturation D4 uptake of all the selected hydrophobic MOFs at $298 \mathrm{~K}$. All CFCMC simulations were carried out for a total of $1 \times 10^{4}$ cycles with $5 \times 10^{3}$ cycles for equilibration. A cycle consists of $\mathrm{N}$ Monte Carlo steps, where $\mathrm{N}$ is equal to the number of molecules (which fluctuates during a CFCMC simulation). For each cycle, random insertion, rotation, translation and continuous-fractional swap moves were attempted. The D4/MOF and $\mathrm{H}_{2} \mathrm{O} / \mathrm{MOF}$ interactions were described by the sum of van der Waals (Lennard-Jones) and Coulombic terms. 
The electrostatic interactions were calculated by the Ewald summation ${ }^{35}$ while a cut-off radius of $12.8 \AA$ was considered for the van der Waals term. Indeed, unit cell dimensions were increased to at least $25.6 \AA$ in each three directions for all MOFs and their frameworks were treated as rigid. Atomic charges for all atoms in the MOFs were estimated using Extended Charge Equilibration (Qeq) method as implemented in RASPA $^{32}$ and their LJ parameters were taken from the UFF forcefield as currently employed ${ }^{36,37}$. H2O was modelled using TIP4P $/ 2005^{38}$. D4 was described by a semi-flexible all atom model with intramolecular parameters taken from the consistent-valence force field (CVFF) ${ }^{39}$ (Tables S1 to S5, SI) while the LJ parameters for all atoms were taken from the UFF forcefield as done in earlier work ${ }^{40}$ and their charges were calculated at the DFT level (Table S6, SI).

All the results of the HCTS are available as CSV files in the SI. A web-based explorer, which can be used to interactively display the dataset is available at https://pauliacomi.com/ mof4d4.

2.2. MOF sorbents. The benchmark MIL-101(Cr) sample was taken from a previous work ${ }^{41}$, with all textural characteristics as stated in reference. DUT-4(Al) was purchased from Materials Center (TU Dresden, Germany). PXRD, TGA and $\mathrm{N}_{2}$ physisorption measurements for DUT-4(AI) are available in the SI (Fig. S6). Brunauer-Emmet-Teller (BET) areas of $3475 \mathrm{~m}^{2} \mathrm{~g}^{-1}$ and $1610 \mathrm{~m}^{2} \mathrm{~g}^{-1}$ were determined for MIL-101(Cr) and DUT-4(Al), respectively. PCN-777 was synthesised by optimizing a previous published methodology ${ }^{42}$. Full synthesis methodology, activation procedure and phase purity analysis using TGA, PXRD and $\mathrm{N}_{2}$ physisorption are given in the $\mathrm{SI}$. All samples were activated at $423 \mathrm{~K}$ under vacuum prior to adsorption experiments.

2.3. Material characterization. $P X R D$ patterns were recorded on a Panalytical X'Pert PRO PXRD diffractometer with a $\mathrm{Cu}$ $K_{\alpha}$ radiation source, in a Bragg-Brentano reflection geometry, using a spinning sample holder with a low-background silicon insert. $\mathrm{N}_{2}$ isotherms at $77 \mathrm{~K}$ were recorded in a Micromeritics Tristar manometric analyser (displayed in Fig. S8, SI). The BET areas were calculated using the pyGAPS suite ${ }^{43}$, with the application of the Rouquerol rules for isotherm region selection yielding a minimum Pearson correlation coefficient of $R=0.997$ (see Fig. S9 for resulting fitting).

2.4. D4 sorption experiments. Sorption measurements were gravimetrically recorded using a dynamic method in a DVS Vacuum instrument (Surface Measurement Systems, UK). In this setup, a continuous adsorbate flow sourced from the headspace of a reservoir enters the sample enclosure, passes the suspended sample pan, and is entrained by a vacuum system. Pressure is controlled by a butterfly valve located before the outlet. Uptake is monitored by a magnetically suspended balance, capable of measuring mass changes at a resolution of $0.1 \mu \mathrm{g}$. The entire apparatus is kept in a temperature-controlled chamber to avoid any condensation points. For each experiment, a stainless-steel sample pan is first tared, then loaded with about $10 \mathrm{mg}$ of sample. The sample is activated in situ under dynamic vacuum $\left(1 \times 10^{-2} \mathrm{~Pa}\right)$ to $423 \mathrm{~K}$. The adsorption-desorption isotherms for $\mathrm{D} 4$ and $\mathrm{H}_{2} \mathrm{O}$ and subsequent repeats were recorded at $303 \mathrm{~K}$ in the $0-10 \mathrm{~Pa}$ range of pressure. Adsorption cycling was similarly recorded, switching between two setpoints of low $(0.5 \mathrm{~Pa})$ and high pressure $(10 \mathrm{~Pa})$. The $\mathrm{D} 4$ used for the sorption experiments was sourced from Sigma Aldrich, with minimum $98 \%$ purity.

\section{Results and discussion}

3.1. Pre-selection of hydrophobic MOFs. We first excluded from our considered MOF database all structures with PLDs lower than $6 \AA$, a threshold selected as the average between the kinetic diameter of D4 (8.6 $\AA)$ and the effective diameter of its constitutive inner $\mathrm{Si}-\mathrm{O}$ ring $(4.5 \AA)$. A total of 1739 remaining non-disordered MOFs were further considered, their geometric and textural properties, i.e. PV, SA, and $\phi$, as well as their density $(\rho)$ being summarized in Fig. S1. As siloxanerich biogas streams often contain water vapour, the optimal D4 adsorbent should have a relatively low water affinity to avoid competing adsorption. Moreover, hydrophobic MOFs are known to possess increased resistance to the hydrolysis of the metal-linker bond ${ }^{44,45}$, alleviating long-term water stability concerns. Therefore, we screened the water affinity of the 1739 MOFs by computing their Henry coefficient of water $\left(K_{\mathrm{H}_{2} \mathrm{H}_{2} \mathrm{O}}\right)$ and the isosteric enthalpy of adsorption at infinite dilution $\left(\Delta H_{s t, H_{2} O}^{0}\right)$ at $298 \mathrm{~K}$ using the Widom particle insertion method $^{33}$. This approach is generally applied in HTCS studies, providing a quick way to gauge the hydrophobicity/hydrophilicity of MOFs ${ }^{36,46}$. All the computational details including the force fields used to describe both MOFs and water are provided in the methodology section and SI. In the frame of biogas upgrading, an extremely hydrophobic adsorbent is not required since the water content usually ranges from $38 \%$ to $85 \%$ relative humidity ${ }^{4}$, therefore the following thresholds were applied to select MOFs with moderate to high hydrophobicity: $K_{H, H_{2} O}<$ $1 \times 10^{-5} \mathrm{~mol} \mathrm{~kg}^{-1} \mathrm{~Pa}^{-1}$ and $\Delta H_{s t, H_{2} O}^{0}<33 \mathrm{~kJ} \mathrm{~mol}^{-1}$ (below the vaporization enthalpy of water $\left.\sim 40 \mathrm{~kJ} \mathrm{~mol}^{-1}\right)^{47}$. As a frame of reference, the highly hydrophobic ZIF-8 is characterized by $K_{H, H_{2} O}=2.5 \times 10^{-6} \mathrm{~mol} \mathrm{~kg}^{-1} \mathrm{~Pa}^{-1}$ and $\Delta H_{s t, H_{2} O}^{0}=$ $30 \mathrm{~kJ} \mathrm{~mol}^{-148}$. Overall, among the $1739 \mathrm{MOFs}, 811$ structures ( $47 \%$ of our material library) were predicted to fulfill these two criteria. This hydrophobic MOF dataset encompasses structures of density ranging from $0.24 \mathrm{~g} \mathrm{~cm}^{-3}$ to $2.04 \mathrm{~g} \mathrm{~cm}^{-3}$ and with a wide range of geometric and textural features: 6

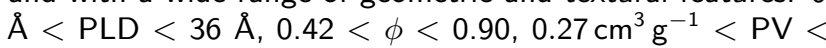
$3.72 \mathrm{~cm}^{3} \mathrm{~g}^{-1}$ and $320 \mathrm{~m}^{2} \mathrm{~g}^{-1}<\mathrm{SA}<6700 \mathrm{~m}^{2} \mathrm{~g}^{-1}$, as shown in Fig. S1.

3.2. Prediction of the D4 uptake performance for the hydrophobic MOFs. As a validation stage of the computational method, the D4 uptakes for MIL-101(Cr) and DUT-4(AI) were first predicted using the CFCMC approach described in the methodology section and compared with the available experimental data. The simulated uptake for MIL-101(Cr), the current best MOF performer, was found to be $1.03 \mathrm{~g} \mathrm{~g}^{-1}$ vs $0.95 \mathrm{~g} \mathrm{~g}^{-1}$ as reported in the original experimental study ${ }^{22}$. We equally confirmed the good agreement between the calculated and the experimental D4 uptake by recording an additional adsorption isotherm on a MIL-101(Cr) sample, finding a D4 capacity of $1.15 \mathrm{~g} \mathrm{~g}^{-1}$ at $298 \mathrm{~K}$. The D4 uptake for DUT-4(AI) was however predicted to be substantially higher $\left(0.42 \mathrm{~g} \mathrm{~g}^{-1}\right)$ than the experimental value reported previously of $0.15 \mathrm{~g} \mathrm{~g}^{-121}$ We therefore collected a D4 adsorption isotherm on a pristine DUT-4(Al) sample, finding a D4 uptake of $0.5 \mathrm{~g} \mathrm{~g}^{-1}$ (Fig. S10) more in line with our theoretical assessment. The lower D4 capacity reported in the original study is attributed to the method used to quantify the adsorbed amount, based on mass loss upon heating. It is likely that only a fraction of D4 was released, since D4 was demonstrated to strongly interact with DUT-4(AI) due to a high confinement in its pores ${ }^{21}$.

Overall, the good agreement between the simulated uptakes and the corresponding experimental data for the previously investigated MOFs served to validate both the applicability of our computational method and the reliability of our experimental setup. This further highlights the importance of a dual 
Table 1: Top 10 promising hydrophobic MOF materials identified for D4 uptake at $298 \mathrm{~K}$.

\begin{tabular}{|c|c|c|c|c|c|c|c|c|c|}
\hline MOF & $\begin{array}{l}\text { PLD } \\
(\AA)\end{array}$ & $\begin{array}{c}\text { SA } \\
\left(\mathrm{m}^{2} \mathrm{~g}^{-1}\right)\end{array}$ & $\begin{array}{c}\rho \\
\left(\mathrm{g} \mathrm{cm}^{-3}\right)\end{array}$ & $\begin{array}{c}\mathrm{PV} \\
\left(\mathrm{cm}^{3} \mathrm{~g}^{-1}\right)\end{array}$ & $\phi$ & $\begin{array}{c}K_{H, H_{2} O} \\
\left(\mathrm{~mol} \mathrm{~kg}^{-1} \mathrm{~Pa}^{-1}\right)\end{array}$ & $\begin{array}{l}\Delta H_{s t, H_{2} O}^{0} \\
\left(\mathrm{~kJ} \mathrm{~mol}^{-1}\right)\end{array}$ & $\begin{array}{l}\text { Gravimetric D4 } \\
\text { uptake }\left(\mathrm{g} \mathrm{g}^{-1}\right)\end{array}$ & $\begin{array}{l}\text { Volumetric D4 } \\
\text { uptake }\left(\mathrm{g} \mathrm{cm}^{-3}\right)\end{array}$ \\
\hline FOTNIN (PCN-777) & 28.36 & 2990 & 0.27 & 3.31 & 0.90 & $2.80 \times 10^{-6}$ & 7.82 & 2.68 & 0.72 \\
\hline RUTNOK & 14.65 & 6200 & 0.24 & 3.72 & 0.90 & $6.70 \times 10^{-6}$ & 14.81 & 2.57 & 0.62 \\
\hline CUSYAR & 12.18 & 5700 & 0.25 & 3.65 & 0.90 & $3.42 \times 10^{-6}$ & 8.15 & 2.35 & 0.59 \\
\hline WUHDAG & 10.50 & 5500 & 0.29 & 2.99 & 0.87 & $4.69 \times 10^{-6}$ & 16.28 & 2.01 & 0.58 \\
\hline HOHMEX & 14.89 & 5000 & 0.32 & 2.74 & 0.87 & $4.66 \times 10^{-6}$ & 13.24 & 1.97 & 0.63 \\
\hline ECOKAJ & 17.58 & 3600 & 0.33 & 2.68 & 0.87 & $6.89 \times 10^{-6}$ & 17.20 & 1.97 & 0.65 \\
\hline DAJWET & 26.59 & 5000 & 0.28 & 3.06 & 0.87 & $7.73 \times 10^{-6}$ & 17.92 & 1.93 & 0.54 \\
\hline RUBDUP & 19.25 & 4200 & 0.30 & 2.90 & 0.87 & $3.79 \times 10^{-6}$ & 11.62 & 1.93 & 0.58 \\
\hline WUHCUZ & 12.21 & 5500 & 0.30 & 2.91 & 0.87 & $3.75 \times 10^{-6}$ & 12.94 & 1.80 & 0.54 \\
\hline ADATAC & 10.28 & 5130 & 0.34 & 2.57 & 0.87 & $4.16 \times 10^{-6}$ & 12.78 & 1.68 & 0.57 \\
\hline
\end{tabular}

experimental-computational approach even prior to starting the high-throughput screening. We then transitioned towards the search for better performers amongst the 811 identified hydrophobic MOFs. Fig. 2a reports their computed D4 uptakes vs. their $\Delta H_{s t, H_{2} O}^{0}$ values at $298 \mathrm{~K}$, with a similar correlation depicted vs. $K_{H, H_{2} O}$ in Fig. S4, SI. The dashed line represents the current known upper bound for D4 uptake in MOFs, considering MIL-101(Cr) as the benchmark sorbent $\left(0.95 \mathrm{~g} \mathrm{~g}^{-1}\right)^{22}$ 56 hydrophobic MOFs were predicted to be more attractive candidates than MIL-101(Cr) on the basis of gravimetric D4 uptake. Common geometric and textural features of these MOF candidates are void fractions $\phi$ larger than 0.81 and pore volumes (PV) higher than $\sim 1.7 \mathrm{~cm}^{3} \mathrm{~g}^{-1}$. Typically, the relation between gravimetric D4 uptake and PV is shown in Fig. S3.

The 10 best MOFs showing the highest D4 uptakes ranging from 1.68 to $2.68 \mathrm{~g} \mathrm{~g}^{-1}$ are highlighted in Fig. 2a by their Cambridge Structural Database (CSD) ${ }^{49}$ refcode and listed in Table 1. Notably, all these identified candidates were found to be highly hydrophobic with associated $K_{\mathrm{H}, \mathrm{H}_{2} \mathrm{O}}$ of about $5 \times 10^{-6} \mathrm{~mol} \mathrm{~kg}^{-1} \mathrm{~Pa}^{-1}$ and their $\Delta H_{s t, H_{2} O}^{0}$ ranging from 8 to $18 \mathrm{~kJ} \mathrm{~mol}^{-1}$ which make these adsorbents also potentially effective under moderate humidity conditions. Table 1 shows that the highly hydrophobic FOTNIN is predicted to exhibit the highest saturated D4 uptake $\left(2.68 \mathrm{~g} \mathrm{~g}^{-1}\right)$, in relation with its high PV $\left(3.31 \mathrm{~cm}^{3} \mathrm{~g}^{-1}\right)$ and large mesoporous cages $(33.7 \AA$ $\times 28.4 \AA$ ). Remarkably, this gravimetric D4 loading translates into a spectacular improvement as compared to MIL-101(Cr ${ }^{22}$. RUTNOK (common name IRMOF- $76^{50}$ ) gave almost a similar D4 uptake $\left(2.57 \mathrm{~g} \mathrm{~g}^{-1}\right)$ as FOTNIN, in part due to similar PV $\left(3.72 \mathrm{~cm}^{3} \mathrm{~g}^{-1}\right)$ and $\phi(0.9)$. Other candidates exhibit high D4 uptakes, including CUSYAR (also known as MOF$210^{51}$ ), WUHDAG and WUHCUZ (NU-1104, and NU-1103 ${ }^{52}$, respectively). Full structural properties of these 10 MOFs including organic ligands and metal sites are given in Table S8.

In the scope of the practical application of a sorbent for a filter bed or column, volumetric uptake is a reliable metric due to its direct relation to equipment sizing. Trade-offs between gravimetric and volumetric uptakes have been previously reported for the storage of various fluids using porous materials ${ }^{26}$ Fig. $2 b$ shows the relation between the computed gravimetric and volumetric D4 uptakes for the hydrophobic MOFs database. Unlike gravimetric uptake which increases indefinitely, the volumetric uptake in porous materials is limited by the density of the adsorbate fluid phase, to which it asymptotically approaches as framework density decreases (and void fraction increases) ${ }^{53}$. Interestingly FOTNIN remains the top MOF performer in terms of volumetric uptake as well $\left(0.72 \mathrm{~g} \mathrm{~cm}^{-3}\right.$, see Fig. 2b). This MOF (common name $\mathrm{PCN}-777^{42}$ ) is built from large planar tritopic linkers $(4,4$ ', 4' '-s-triazine-2,4,6-triyltri-benzoate or TATB) coordinated to $\mathrm{Zr}_{6}$-oxoclusters in an antiprismatic fashion, forming vertex-sharing supertetrahedra surrounding a mesoporous cage of $33.7 \AA$ as depicted in Fig. 2c These cages are interconnected by hexagonal windows (30 $\AA$ ) and are typically decorated by $\mathrm{OH} / \mathrm{H}_{2} \mathrm{O}$ moieties coordinated to the remaining axial positions of the $\mathrm{Zr}_{6}$ node.

3.3. Experimental assessment of the D4 sorption performance for the top MOF. While HTCS enabled a rapid and effective screening on the performance indicator, additional criteria, such as thermal/chemical stability, synthesis route, activation conditions, precursor toxicity and linker availability need to be considered to select the optimal adsorbents. We therefore critically assessed PCN-777 prior to further experimental action. Our selection criteria for $\mathrm{PCN}-777$ were (i) the
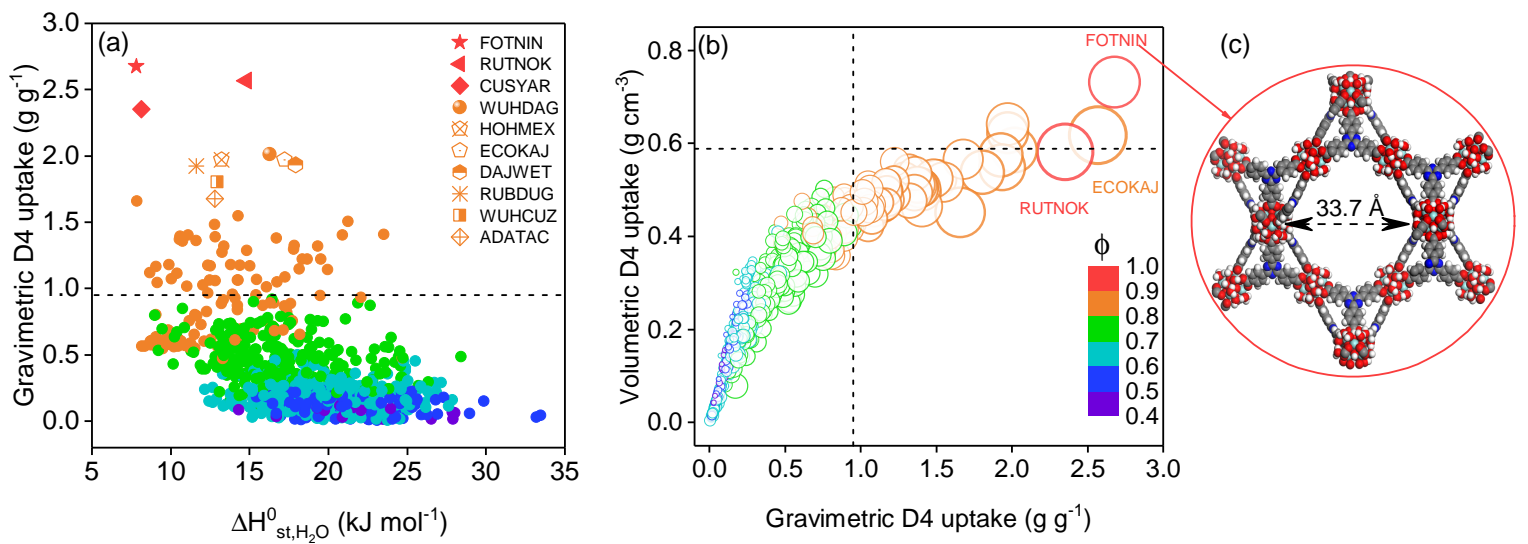

Fig. 2: (a) Predicted D4 uptake performance at $298 \mathrm{~K}$ for the hydrophobic MOF database plotted as a function of computed $\Delta H_{s t, H_{2} O}^{0}$, and colour coded by void fraction, $\phi$. Top performing 10 candidates are represented by different symbols in the legend. (b) Relation between gravimetric ( $\mathrm{g}^{-1}$ ) and volumetric $\left(\mathrm{g} \mathrm{cm}^{-3}\right)$ D4 uptake for all MOFs at $298 \mathrm{~K}$. Marker size represents PV while colour denotes $\phi$. Dashed line represents the gravimetric and volumetric uptake of benchmark MIL-101(Cr) ${ }^{22}$. (c) Illustration of the structure of our promising material identified for D4 uptake, PCN-777. Zr, N, O, C, and H atoms are depicted in light blue, dark blue, red, dark grey, and light grey, respectively. 

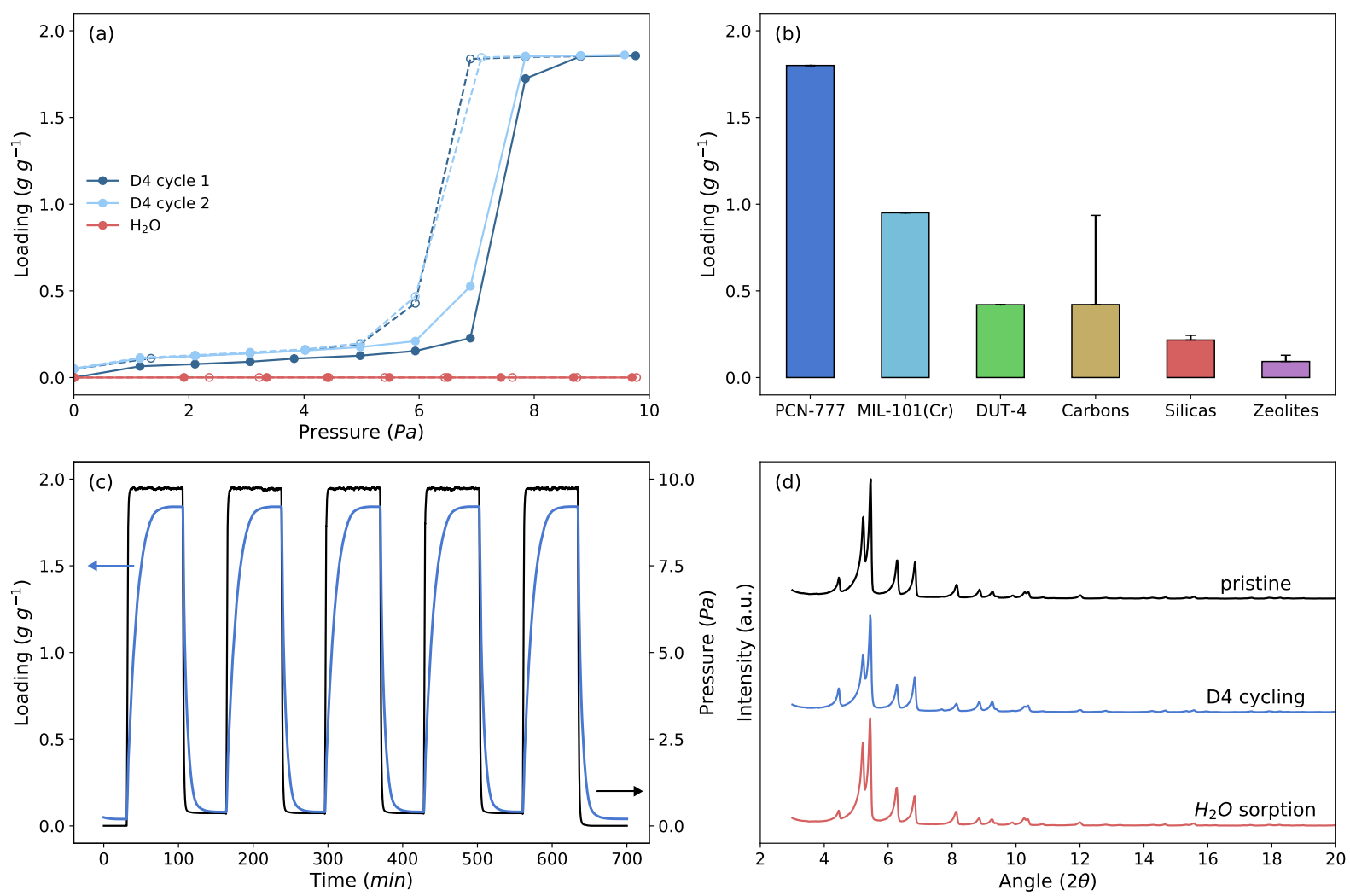

Fig. 3: (a) Single component adsorption/desorption isotherms for $\mathrm{D} 4$ (blue) and $\mathrm{H}_{2} \mathrm{O}$ (red) collected at $303 \mathrm{~K}$ for $\mathrm{PCN}-777$ in the pressure range of $0-10 \mathrm{~Pa}$ (corresponding to $0-0.05 \mathrm{p} / \mathrm{p}^{0}$ for D4). Solid and open symbols represent adsorption and desorption branches, respectively. (b) Comparison of the D4 capacity of MOFs investigated in the present study with other classes of porous materials (data from Wang et al. ${ }^{4}$ ), with error bars placed at one standard deviation of mean capacity. (c) 5 D4 sorption-desorption cycles recorded after the first two isotherms on PCN-777, in the same pressure range. (d) PXRD of pristine PCN-777 sample (black) and samples recovered after D4 cycling (blue) and water adsorption (red)

excellent known stability of the oxo-Zr-carboxylate metal node, at the origin of the high chemical and thermal resistance of the framework, alongside with (ii) the commercially available linker and well-controlled synthesis procedure documented elsewhere $^{42,54}$. Indeed, this material was synthesised accordingly (details provided in the methodology section).

The D4 adsorption isotherm for PCN-777 was first recorded up to $10 \mathrm{~Pa}$ at $303 \mathrm{~K}$ using a dynamic vapour sorption system (experimental details in the methodology section). The resulting isotherm, depicted in Fig. 3a, exhibits a characteristic type $\mathrm{V}$ shape ${ }^{55}$ with a sharp D4 uptake increase above $7 \mathrm{~Pa}$ up to a maximum of $1.8 \mathrm{~g} \mathrm{~g}^{-1}$ that translates into $0.49 \mathrm{~g} \mathrm{~cm}^{-3}$ This value is however lower than the predicted uptake due to two combined reasons: (i) an incomplete evacuation of the porosity (theoretical $\mathrm{PV}=3.3 \mathrm{~cm}^{3} \mathrm{~g}^{-1}$ vs the experimental one of $2.2 \mathrm{~cm}^{3} \mathrm{~g}^{-1}$ determined through $\mathrm{N}_{2}$ physisorption at $77 \mathrm{~K}$, in Fig. S8, SI) commonly observed for mesoporous MOFs ${ }^{56,57}$ and (ii) only a partial accessibility of the super-tetrahedral cages to D4 owing to their relatively small windows. Indeed, while optimized activation procedures may recover more of the expected porosity, the attained D4 uptake constitutes a record among porous solids. This positions $\mathrm{PCN}-777$ as the crystalline porous material with the highest currently known D4 uptake, almost twice higher than the benchmark MIL-101(Cr), 5-10 times that of the most promising silicas and zeolites, and above the best performing activated carbons as illustrated in Fig. $3 b^{4}$. Notably, the step-like adsorption behaviour is ideal from the application point of view of a breakthrough filter, as it ensures a narrow mass transfer zone and minimises the column dead zone at break point. Remarkably, the maximum uptake for PCN-777 is attained at low pressure of $7 \mathrm{~Pa}$ that makes this MOF highly promising for D4 removal in a gas phase concentration below 75 ppm.
Throughout desorption (dotted line with open symbols in Fig. 3a), a small hysteresis occurs with a width of about $1 \mathrm{~Pa}$. Under complete vacuum, a minute amount of D4, about $0.1 \mathrm{~g} \mathrm{~g}^{-1}$, i.e. $5 \%$ of total capacity, is retained in the structure. We attribute this capacity loss to D4 molecules irreversibly trapped in the super-tetrahedral cages or on a small fraction of defect sites. Overall, PCN-777 acts as a highly reversible D4-adsorbent. A second sorption cycle reveals the excellent repeatability of D4 sorption by this MOF, with identical condensation pressure and total uptake, the adsorptiondesorption branches now overlapping in the very low-pressure region (Fig. 3a).

To further investigate the D4 adsorption-desorption cyclability of PCN-777, a subsequent set of five cycles was recorded on the same sample, covering the entire uptake range from fully loaded to empty under a medium vacuum level of $0.5 \mathrm{~Pa}$ (see Fig. 3c). No further capacity loss is observed after the initial $5 \mathrm{wt} \%$ from cycle 1 to cycle 2 with a pressure drop sufficient to fully remove adsorbed D4 in every cycle without the need of thermal treatment. This is a leap forward compared to the previous MOF candidates, i.e. MIL-101(Cr) and DUT-4(Al) The former was reported ${ }^{22}$ to be fully regenerable only at high temperatures (outgassed under vacuum at $423 \mathrm{~K}$ ), and we note that vacuum alone was unable to fully desorb D4, with nearly $50 \%$ of siloxane remaining in the structure after desorption in our experiments (Fig. S10, SI). D4 adsorption in DUT-4(AI) is even more irreversible, owing to the strong confinement of the siloxane molecules in its pores ${ }^{21}$, with essentially no desorption observed under vacuum (Fig. S10, SI). The global sorption kinetics was further qualitatively evaluated by observing the equilibration time throughout cycling steps. Fig. 3c reveals that an adsorption/desorption cycle can be achieved in less than 30 minutes. Such a fast kinetics is a clear advantage 


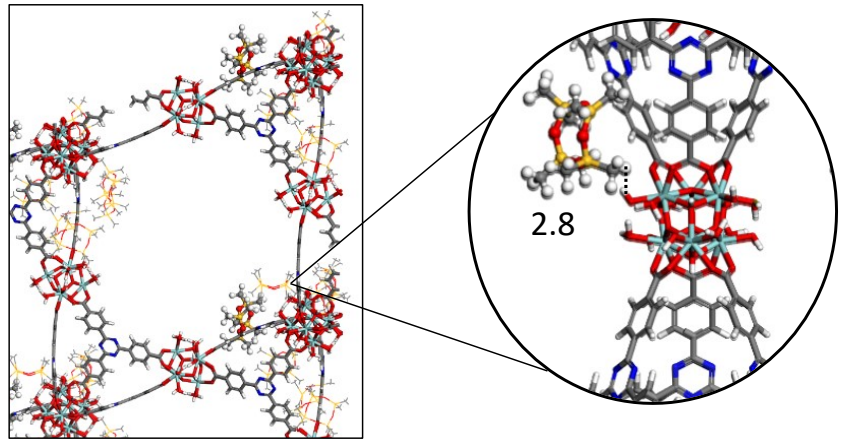

(b) $\theta: 40 \%$

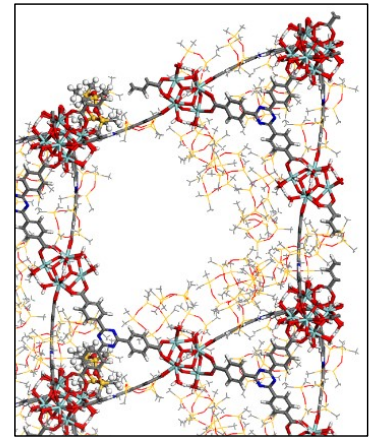

(c) $\theta: 65 \%$

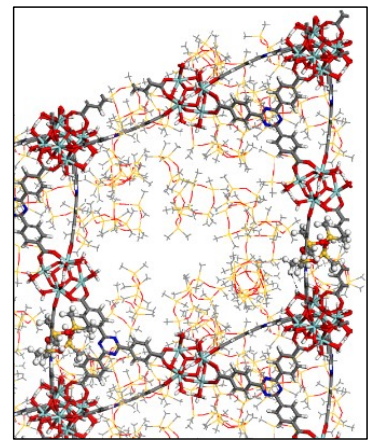

(d) $\Theta: 100 \%$

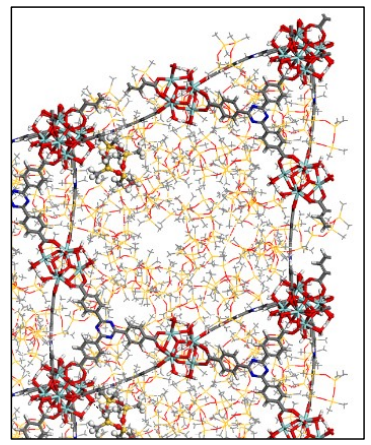

Fig. 4: Representative snapshots of the preferential sitting of D4 in the pores of PCN- 777 at $298 \mathrm{~K}$ for increasing loading at (a) $10 \%$ with highlighted interactions distance between D4 and the MOF framework, and at (b) $40 \%$, (c) $65 \%$, and (d) $100 \%$ fractional loading ( $\theta$ ). Framework atoms (sticks) and D4 molecules (lines, and ball and sticks) are coded as $\mathrm{Zr}, \mathrm{N}, \mathrm{O}, \mathrm{Si}, \mathrm{C}$, and $\mathrm{H}$ atoms in light blue, dark blue, red, yellow, dark grey, and light grey respectively. The separating distance is represented by dashed black lines and reported in $\AA$

for practical use. In addition, the water adsorption collected for PCN-777 further confirmed its predicted hydrophobicity and revealed that below $P=7 \mathrm{~Pa}$, water loading is negligible, i.e. under $0.02 \mathrm{~g} \mathrm{~g}^{-1}$ (see Fig. 3a). This observation strongly suggests that PCN-777 is expected to maintain its high-level performance for D4 removal under low to moderate humidity working conditions.

Stability of PCN-777 after its use as a D4 adsorbent was also evaluated by checking its crystallinity and porosity. PXRD patterns recorded after the D4 cycling experiments show similar Bragg peak positions and broadenings as the pristine material, testifying that no amorphisation or decrease of crystallinity were incurred (Fig. 3d). The same conclusion holds true for PCN777 upon water adsorption. Further, $\mathrm{N}_{2}$ adsorption isotherms collected at $77 \mathrm{~K}$ for PCN-777 after $\mathrm{H}_{2} \mathrm{O}$ and D4 adsorption both present a similar shape than that of the pristine solid (see Fig. S8). Slightly lower pore volume $\left(1.87 \mathrm{~cm}^{3} \mathrm{~g}^{-1}\right.$ vs $\left.2.20 \mathrm{~cm}^{3} \mathrm{~g}^{-1}\right)$ and BET area $\left(1544 \mathrm{~m}^{2} \mathrm{~g}^{-1}\right.$ vs $\left.1730 \mathrm{~m}^{2} \mathrm{~g}^{-1}\right)$ were obtained for the material after D4 cycling compared to the pristine solid, attributed to the small amount of D4 retained in the porous framework during the first adsorption cycle.

3.4. Adsorption mechanism. A careful analysis of the adsorption mechanism of D4 in PCN-777 was further explored by considering $\mathrm{MC}$ simulations in the canonical ensemble with increasing loading up to the saturation. At the initial stage of adsorption, the coordinated $\mathrm{OH} / \mathrm{H}_{2} \mathrm{O}$ moieties of the MOF $\mathrm{Zr}_{6}$ node pointing towards the pore were found to act as primary adsorption sites (Fig. 4a). The D4 molecule interacts mostly via its methyl group with an averaged separating $\mathrm{H}\left(\mathrm{CH}_{3}\right)-\mathrm{H}\left(\mathrm{H}_{2} \mathrm{O}\right)$ distance of $2.8 \AA$ (see the radial distribution function plotted for the corresponding pair in Fig. S5a) as illustrated in Fig. 4a. This preferential sitting of D4 is associated with a moderately high simulated adsorption enthalpy of $83.5 \mathrm{~kJ} \mathrm{~mol}^{-1}$ in line with the isosteric heat of adsorption we assessed experimentally that ranges from 65 and $75 \mathrm{~kJ} \mathrm{~mol}^{-1}$ (Fig. S11). Both values are higher than the enthalpy of liquefaction of D4 at $303 \mathrm{~K}$ as $54.5 \mathrm{~kJ} \mathrm{~mol}^{-147}$. We further demonstrated that this value remains substantially lower than the one simulated for DUT-4(AI) $\left(194.0 \mathrm{~kJ} \mathrm{~mol}^{-1}\right)$ for which the adsorption of D4 is governed by a high degree of confinement leading to an irreversible process. This observation clearly states that the adsorption energetics in PCN-777 offers a good compromise to ensure an efficient adsorption of D4 as well as an almost fully reversible and fast adsorption/desorption process. While increasing the loading, D4 molecules tend to form a monolayer near the wall of the cage owing to their interactions with both the organic linkers and inorganic nodes of the MOF as shown in Fig. 4b-c. Finally, at higher loading, the molecules form multilayers and further occupy the whole cage corresponding to the scenario of the capillary condensation (Fig. 4d). This effective packing is governed by guest-guest interactions involving averaged separating $\mathrm{H}\left(\mathrm{CH}_{3}\right)-\mathrm{H}\left(\mathrm{CH}_{3}\right)$ distance of $2.7 \AA$ at saturation (the radial distribution function plotted for this pair is shown in Fig. S5b). Such pore filling mechanism has been commonly observed in diverse mesoporous materials for a range of molecules ${ }^{58}$. Indeed, PCN-777 exhibits an ideal combination of a large cage to enable an effective packing of the siloxane molecules and the presence of moieties accessible to D4 to favour moderately high host/guest interactions to ensure an efficient trapping of the D4 molecules initially adsorbed.

\section{Conclusions}

In this work, a high throughput computational screening first identified a series of hydrophobic MOFs with octamethylcyclotetrasiloxane uptakes outperforming by far the performance of the conventional adsorbents. The best-predicted MOF performer, PCN-777, was synthesized and its predicted exceptional 

adsorption capacity for this typical contaminant present in bio-

\section{Contributions}

\section{${ }_{498}$ Conflicts of interest} gas was further experimentally confirmed. This stable MOF was demonstrated to exhibit record gravimetric $\left(1.8 \mathrm{~g} \mathrm{~g}^{-1}\right)$ and volumetric $\left(0.49 \mathrm{~g} \mathrm{~cm}^{-3}\right)$ uptake alongside with a reversible and fast adsorption/desorption process, very good cyclability and easy regeneration under continuous pressure cycling owing to a step-like sorption isotherm. The attractiveness of PCN-777 was found to result from a synergistic combination of mesoporous cages and chemical functionality pointing towards the center of the cages to ensure moderately high host/guest interactions and favour an efficient removal of D4 at low pressure and an efficient packing of the siloxane molecules at higher pressure while maintaining the process highly reversible. Moreover, its hydrophobicity makes this MOF promising for the selective removal of siloxanes in moderate humidity conditions. In a broader sense, this study highlights the efficacy of an integrated workflow for accelerating the selection of adsorbents for a target application, spanning the entire pipeline from method validation to computational screening, synthesis, adsorption testing and finally identification of the optimal candidates.

\section{Acknowledgements}

We thank Centre National d'Etudes Spatiales for research funding (R\&T n ${ }^{\circ}$ R-S20/MT-0002-03 and PhD). PXRD experiments were performed with the support of the Balard Plateforme d'Analyses et de Caractérisation (PAC Balard).

Conceptualization: EG, PI, GR, SDV, GM. Computational investigation, methodology, data analysis: EG, and GM. Screening data curation: EG. Visualization: PI. PCN-777 synthesis: YK and JSK. Experimental investigation, methodology, data analysis: PI and SDV. Writing - original draft: EG, PI, SDV, GM. Writing - review and editing: all authors. Supervision: JSK, SDV, GM.

There are no conflicts of interest to declare.

\section{References}

1. Themelis, N.J. and Ulloa, P.A. "Methane generation in landfills." Renewable Energy, 2007. 32(7):1243-1257. doi:10.1016/j.renene.2006.04.020

2. Takuwa, Y.; Matsumoto, T.; Oshita, K.; Takaoka, M.; Morisawa, S.; and Takeda, N. "Characterization of trace constituents in landfill gas and a comparison of sites in Asia." J Mater Cycles Waste Manag, 2009. 11(4):305-311. doi:10.1007/s10163-009-0257-1

3. Ohannessian, A.; Desjardin, V.; Chatain, V.; and Germain, P. "Volatile organic silicon compounds: The most undesirable contaminants in biogases." Water Science and Technology, 2008. 58(9):1775-1781. doi:10.2166/wst.2008.498

4. Wang, G.; Zhang, Z.; and Hao, Z. "Recent advances in technologies for the removal of volatile methylsiloxanes: A case in biogas purification process." Critical Reviews in Environmental Science and Technology, 2019. 49(24):2257-2313. doi:10.1080/10643389.2019.1607443

5. Dewil, R.; Appels, L.; and Baeyens, J. "Energy use of biogas hampered by the presence of siloxanes." Energy Conversion and Management, 2006. 47(13-14):1711-1722. doi:10.1016/j.enconman.2005.10.016

6. Kuhn, J.N.; Elwell, A.C.; Elsayed, N.H.; and Joseph, B. "Requirements, techniques, and costs for contaminant removal from landfill gas." Waste Management, 2017 63:246-256. doi:10.1016/j.wasman.2017.02.001

7. Chin, K.F.; Wan, C.; Li, Y.; Alaimo, C.P.; Green, P.G.; Young, T.M.; and Kleeman, M.J. "Statistical analysis of trace contaminants measured in biogas." Sci Total Environ, 2020. 729:138702

8. Ajhar, M.; Travesset, M.; Yüce, S.; and Melin, T. "Siloxane removal from landfill and digester gas - a technology overview." Bioresour Technol, 2010. 101(9):2913-2923

9. Finocchio, E.; Garuti, G.; Baldi, M.; and Busca, G. "Decomposition of hexamethylcyclotrisiloxane over solid oxides." Chemosphere, 2008. 72(11):1659-1663

10. Montanari, T.; Finocchio, E.; Bozzano, I.; Garuti, G.; Giordano, A.; Pistarino, C.; and Busca, G. "Purification of landfill biogases from siloxanes by adsorption: A study of silica and 13X zeolite adsorbents on hexamethylcyclotrisiloxane separation." Chemical Engineering Journal, 2010. 165(3):859-863. doi:10.1016/j.cej.2010.10.032

11. Sigot, L.; Ducom, G.; and Germain, P. "Adsorption of octamethylcyclotetrasiloxane (D4) on silica gel (SG): Retention mechanism." Microporous and Mesoporous Materials, 2015. 213:118-124. doi:10.1016/j.micromeso.2015.04.016

12. Schweigkofler, M. and Niessner, R. "Removal of siloxanes in biogases." Journal of Hazardous Materials, 2001. 83(3):183-196. doi:10.1016/S0304-3894(00)00318-6
13. Férey, G. "Hybrid porous solids: Past, present, future." Chem Soc Rev, 2008 37(1):191-214. doi:10.1039/B618320B

14. Zhou, H.C.; Long, J.R.; and Yaghi, O.M. "Introduction to Metal-Organic Frameworks." Chemical Reviews, 2012. 112(2):673-674. doi:10.1021/cr300014×

15. Evans, J.D.; Bon, V.; Senkovska, I.; Lee, H.C.; and Kaskel, S. "Four-dimensional metal-organic frameworks." Nat Commun, 2020. 11(1):2690. doi:10.1038/s41467020-16527-8

16. Siegelman, R.L.; Milner, P.J.; Kim, E.J.; Weston, S.C.; and Long, J.R. “Challenges and opportunities for adsorption-based $\mathrm{CO}_{2}$ capture from natural gas combined cycle emissions." Energy Environ Sci, 2019. 12(7):2161-2173. doi:10.1039/C9EE00505F

17. Lin, R.B.; Xiang, S.; Zhou, W.; and Chen, B. "Microporous MetalOrganic Framework Materials for Gas Separation." Chem, 2020. 6(2):337-363. doi:10.1016/j.chempr.2019.10.012

18. Bavykina, A.; Kolobov, N.; Khan, I.S.; Bau, J.A.; Ramirez, A.; and Gascon, J. "Metal-Organic Frameworks in Heterogeneous Catalysis: Recent Progress, New Trends, and Future Perspectives." Chem Rev, 2020. 120(16):8468-8535. doi:10.1021/acs.chemrev.9b00685

19. Allendorf, M.D.; Dong, R.; Feng, X.; Kaskel, S.; Matoga, D.; and Stavila, V. "Electronic Devices Using Open Framework Materials." Chem Rev, 2020. 120(16):85818640. doi:10.1021/acs.chemrev.0c00033

20. Woellner, M.; Hausdorf, S.; Klein, N.; Mueller, P.; Smith, M.W.; and Kaskel, S. "Adsorption and Detection of Hazardous Trace Gases by Metal-Organic Frameworks." Adv Mater, 2018. 30(37):1704679. doi:10.1002/adma.201704679

21. Mito-oka, Y.; Horike, S.; Nishitani, Y.; Masumori, T.; Inukai, M.; Hijikata, Y.; and Kitagawa, S. "Siloxane D4 capture by hydrophobic microporous materials." J Mater Chem A, 2013. 1(27):7885. doi: $10.1039 /$ c3ta11217a

22. Gargiulo, N.; Peluso, A.; Aprea, P.; Marino, O.; Cioffi, R.; Jannelli, E.; Cimino, S. Lisi, L.; and Caputo, D. "Chromium-based MIL-101 metal organic framework as a fully regenerable D4 adsorbent for biogas purification." Renewable Energy, 2019. 138:230-235. doi:10.1016/j.renene.2019.01.096

23. Zhao, H.; Li, Q.; Wang, Z.; Wu, T.; and Zhang, M. "Synthesis of MIL-101(Cr) and its water adsorption performance." Microporous and Mesoporous Materials, 2020 297:110044. doi:10.1016/j.micromeso.2020.110044

24. Simon, C.M.; Mercado, R.; Schnell, S.K.; Smit, B.; and Haranczyk, M. "What Are the Best Materials To Separate a Xenon/Krypton Mixture?" Chem Mater, 2015. 27(12):4459-4475. doi:10.1021/acs.chemmater.5b01475

25. Park, J.; Lively, R.P.; and Sholl, D.S. "Establishing upper bounds on $\mathrm{CO}_{2}$ swing capacity in sub-ambient pressure swing adsorption via molecular simulation of metal-organic frameworks." J Mater Chem A, 2017. 5(24):12258-12265. doi:10.1039/C7TA02916K

26. Moghadam, P.Z.; Islamoglu, T.; Goswami, S.; Exley, J.; Fantham, M.; Kaminski, C.F.; Snurr, R.Q.; Farha, O.K.; and Fairen-Jimenez, D. "Computer-aided discovery of a metal-organic framework with superior oxygen uptake." Nat Commun, 2018 9(1):1378. doi:10.1038/s41467-018-03892-8

27. Boyd, P.G.; Chidambaram, A.; García-Díez, E.; Ireland, C.P.; Daff, T.D.; Bounds, R.; Gładysiak, A.; Schouwink, P.; Moosavi, S.M.; Maroto-Valer, M.M.; Reimer, J.A. Navarro, J.A.R.; Woo, T.K.; Garcia, S.; Stylianou, K.C.; and Smit, B. "Data-driven design of metal-organic frameworks for wet flue gas CO2 capture." Nature, 2019. 576(7786):253-256. doi:10.1038/s41586-019-1798-7

28. Shi, Z.; Liang, H.; Yang, W.; Liu, J.; Liu, Z.; and Qiao, Z. “Machine learning and in silico discovery of metal-organic frameworks: Methanol as a working fluid in adsorption-driven heat pumps and chillers." Chemical Engineering Science, 2020 214:115430. doi:10.1016/j.ces.2019.115430

29. Yao, Z.; Sánchez-Lengeling, B.; Bobbitt, N.S.; Bucior, B.J.; Kumar, S.G.H.; Collins, S.P.; Burns, T.; Woo, T.K.; Farha, O.K.; Snurr, R.Q.; and Aspuru-Guzik, A. "Inverse design of nanoporous crystalline reticular materials with deep generative models." Nat Mach Intell, 2021. doi:10.1038/s42256-020-00271-1

30. Chung, Y.G.; Haldoupis, E.; Bucior, B.J.; Haranczyk, M.; Lee, S.; Zhang, H. Vogiatzis, K.D.; Milisavljevic, M.; Ling, S.; and Camp, J.S. "Advances, updates, and analytics for the computation-ready, experimental Metal-Organic framework database: CoRE MOF 2019." J Chem Eng Data, 2019. 64(12):5985-5998

31. Willems, T.F.; Rycroft, C.H. Kazi, M. Meza, J.C.; and Haranczyk, M. "Algorithms and tools for high-throughput geometry-based analysis of crystalline porous materials." Microporous and Mesoporous Materials, 2012. 149(1):134-141. doi:10.1016/j.micromeso.2011.08.020

32. Dubbeldam, D.; Calero, S.; Ellis, D.E.; and Snurr, R.Q. "RASPA: Molecular simulation software for adsorption and diffusion in flexible nanoporous materials." Mol Simul, 2016. 42(2):81-101

33. Frenkel, D. and Smit, B. Understanding Molecular Simulation: From Algorithms to Applications, vol. 1. Academic press: London, 2002. ISBN 0-08-051998-9

34. Rahbari, A.; Hens, R.; Ramdin, M.; Moultos, O.A.; Dubbeldam, D.; and Vlugt, T.J.H. "Recent advances in the continuous fractional component Monte Carlo methodology." Molecular Simulation, 2020. pp. 1-20. doi:10.1080/08927022.2020.1828585

35. Ewald, P.P. "Die berechnung optischer und elektrostatischer gitterpotentiale." Ann Phys, 1921. 369(3):253-287

36. Qiao, Z.; Xu, Q.; Cheetham, A.K.; and Jiang, J. "High-throughput computationa screening of metal-organic frameworks for thiol capture." J Phys Chem C, 2017. 121(40):22208-22215

37. Keskin, S.; Liu, J.; Rankin, R.B.; Johnson, J.K.; and Sholl, D.S. "Progress, Opportunities, and Challenges for Applying Atomically Detailed Modeling to Molecular Adsorption and Transport in Metal-Organic Framework Materials." Ind Eng Chem Res, 2009. 48(5):2355-2371. doi:10.1021/ie800666s

38. Abascal, J.L. and Vega, C. "A general purpose model for the condensed phases of water: TIP4P/2005." J Chem Phys, 2005. 123(23):234505-234517

39. Dauber-Osguthorpe, P.; Roberts, V.A.; Osguthorpe, D.J.; Wolff, J.; Genest, M.; and Hagler, A.T. "Structure and energetics of ligand binding to proteins: Escherichia coli dihydrofolate Reductase-Trimethoprim, a Drug-Receptor system." Proteins: Struct, Funct, Bioinf, 1988. 4(1):31-47

40. Xu, R.G. and Leng, Y. "Solvation force simulations in atomic force microscopy." The Journal of Chemical Physics, 2014. 140(21):214702. doi:10.1063/1.4879657

41. Pillai, R.S.; Yoon, J.W.; Lee, S.J.; Hwang, Y.K.; Bae, Y.S.; Chang, J.S.; and Maurin, G. " $\mathrm{N}_{2}$ Capture Performances of the Hybrid Porous MIL-101(Cr): From Prediction toward Experimental Testing." J Phys Chem C, 2017. 121(40):22130 
22138. doi:10.1021/acs.jpcc.7b07029

42. Feng, D.; Wang, K.; Su, J.; Liu, T.F.; Park, J.; Wei, Z.; Bosch, M.; Yakovenko, A. Zou, X.; and Zhou, H.C. "A Highly Stable Zeotype Mesoporous Zirconium MetalOrganic Framework with Ultralarge Pores." Angew Chem Int Ed, 2015. 54(1):149-154. doi:10.1002/anie.201409334

43. lacomi, P. and Llewellyn, P.L. "pyGAPS: A Python-based framework for adsorption isotherm processing and material characterisation." Adsorption, 2019. 25(8):15331542. doi:10.1007/s10450-019-00168-5

44. Burtch, N.C.; Jasuja, H.; and Walton, K.S. "Water Stability and Adsorption in Metal-Organic Frameworks." Chemical Reviews, 2014. 114(20):10575-10612. doi: $10.1021 /$ cr5002589

45. Wu, T.; Shen, L.; Luebbers, M.; Hu, C.; Chen, Q.; Ni, Z.; and Masel, R.I. "Enhancing the stability of metal-organic frameworks in humid air by incorporating water repellent functional groups." Chem Commun, 2010. 46(33):6120-6122

46. Matito-Martos, I.; Moghadam, P.Z.; Li, A.; Colombo, V.; Navarro, J.A.R.; Calero, S.; and Fairen-Jimenez, D. "Discovery of an Optimal Porous Crystalline Material for the Capture of Chemical Warfare Agents." Chem Mater, 2018. 30(14):4571-4579. doi:10.1021/acs.chemmater.8b00843

47. Lemmon, E.W.; Bell, I.; Huber, M.L.; and McLinden, M.O. "NIST standard reference database 23: Reference fluid thermodynamic and transport properties-refprop, version 10.0, national institute of standards and technology." 2018

48. Moghadam, P.Z.; Fairen-Jimenez, D.; and Snurr, R.Q. "Efficient identification of hydrophobic MOFs: Application in the capture of toxic industrial chemicals." J Mater Chem A, 2016. 4(2):529-536

49. Allen, F.H. "The cambridge structural database: A quarter of a million crystal structures and rising." Acta Crystallogr, Sect B: Struct Sci, 2002. 58(3):380-388

50. Oisaki, K.; Li, Q.; Furukawa, H.; Czaja, A.U.; and Yaghi, O.M. "A Metal-organic framework with covalently bound organometallic complexes." J Am Chem Soc, 2010. 132(27):9262-9264

51. Furukawa, H.; Ko, N.; Go, Y.B.; Aratani, N.; Choi, S.B.; Choi, E.; Yazaydin, A.O.; Snurr, R.Q.; O'Keeffe, M.; Kim, J.; and Yaghi, O.M. "Ultrahigh Porosity in Metal-Organic Frameworks." Science, 2010. 329(5990):424-428. doi:10.1126/science. 1192160

52. Wang, T.C.; Bury, W.; Gómez-Gualdrón, D.A.; Vermeulen, N.A.; Mondloch, J.E. Deria, P.; Zhang, K.; Moghadam, P.Z.; Sarjeant, A.A.; and Snurr, R.Q. "Ultrahigh surface area zirconium MOFs and insights into the applicability of the BET theory." J Am Chem Soc, 2015. 137(10):3585-3591

53. Bobbitt, N.S.; Chen, J.; and Snurr, R.Q. "High-throughput screening of Metal-Organic frameworks for hydrogen storage at cryogenic temperature." J Phys Chem C, 2016. 120(48):27328-27341

54. Liu, H.; Xu, C.; Li, D.; and Jiang, H.L. "Photocatalytic hydrogen production coupled with selective benzylamine oxidation over MOF composites." Angew Chem, 2018. 130(19):5477-5481

55. Thommes, M.; Kaneko, K.; Neimark, A.V.; Olivier, J.P.; Rodriguez-Reinoso, F.; Rouquerol, J.; and Sing, K.S.W. "Physisorption of gases, with special reference to the evaluation of surface area and pore size distribution (IUPAC Technical Report)." Pure and Applied Chemistry, 2015. 87(9-10):1051-1069. doi:10.1515/pac-2014-1117

56. Nelson, A.P.; Farha, O.K.; Mulfort, K.L.; and Hupp, J.T. "Supercritical Processing as a Route to High Internal Surface Areas and Permanent Microporosity in MetalOrganic Framework Materials." Journal of the American Chemical Society, 2009. 131(2):458-460. doi:10.1021/ja808853q

57. Park, Y.K.; Choi, S.B.; Kim, H.; Kim, K.; Won, B.H.; Choi, K.; Choi, J.S.; Ahn, W.S.; Won, N.; and Kim, S. "Crystal structure and guest uptake of a mesoporous metal-organic framework containing cages of 3.9 and $4.7 \mathrm{~nm}$ in diameter." Angew Chem, Int Ed, 2007. 46(43):8230-8233

58. Rouquerol, J.; Rouquerol, F.; Llewellyn, P.L.; Maurin, G.; and Sing, K. Adsorption by Powders and Porous Solids: Principles, Methodology and Applications. 2013. ISBN 978-0-08-097035-6 